\section{S-229 MECHANISTIC EVIDENCE IN POPULATION STUDIES ON NIGHT SHIFT AND CANCER}

'Manolis Kogevinas. 'IS GLOBAL, Spain

10.1136/OEM-2021-EPI.423

Introduction In 2007 IARC classified night-shift work as probably carcinogenic to humans (2A). A new evaluation in 2019, 12 years later, resulted in a similar conclusion. It was problematic that numerous cohort studies had poor exposure assessment and population studies examining key characteristics (KCs) of carcinogens were scarce and frequently small.

Objectives I will first summarise molecular epidemiology studies and identify problems that have hampered the interpretation of $\mathrm{KC}$ regarding night shift and cancer. I will then discuss strategies for future studies and focus on a new exposome protocol in night shift workers in Europe (EPHOR study).

Methods/Results In the EPHOR study, we will collect individual data and repeated biological samples in 800 night and day shift workers (Spain, Sweden, Denmark, The Netherlands). Exposures of interest measured mostly with personal sensors include light and light spectrum, physical activity, sleep, body temperature, noise, the timing of activities, and some chemical exposures. We will evaluate biomarkers related to immune response, inflammation, disruption of sex-steroid hormones, melatonin and cortisol, cardiometabolic status, markers of aging e.g. telomere attrition together with untargeted omics analyses. Other outcomes of interest include obesity, diabetes, and cognitive and mental outcomes.

Conclusions Some of the endpoints examined are KCs of carcinogens, others define the circadian pattern (e.g. melatonin) and others are important for non-cancer endpoints. The circadian patterns of several markers complicate interpretation since differences between day and night working hours may reflect normal variation. Interpretation of changes in time of peak of a marker (e.g. testosterone) is also complex since on some occasions this may affect functions while in others may just indicate adaptation to a changed schedule. With its large size, standardized procedures, and multiple endpoints examined including several KCs of carcinogens, we expect that the EPHOR study should provide new knowledge on the health effects related.

\section{S-234 STRATEGIES FOR MONITORING OF THE INTERNAL EXPOSOME USING SELF-SAMPLING METHODS IN THE CONTEXT OF EU EPHOR PROJECT}

${ }^{1}$ Eline Verscheure, Vivi Schlünssen, Manolis Kogevinas, Barbara Harding, Susan Peters, Roel Vermeulen, Rob Stierum, Anjoeka Pronk, Lode Godderis, Manosij Ghosh, William Murch, Anne Mette Lund Würtz, Karin Broberg, Shan Zienolddiny, Johanna Samulin-Erdem. 'KU Leuven, Belgium

\subsection{6/OEM-2021-EPI.424}

Introduction Working life exposures contribute significantly to non-communicable disease development. However, the challenge remains on how to map occupational exposures during the entire career and link exposures with health outcomes. In this context, the EU EPHOR project aims to characterize the internal exposome, by characterizing exposure biomarkers and biological pathways to link external exposure and health effects. While there is a range of strategies available to monitor the internal exposome, these conventional methods often require invasive collection of biological samples and/or high volumes. However, the ongoing COVID-19 pandemic forces us to look also at other approaches to obtain biological samples.

Objective We aimed to explore the use of self-sampling techniques in an occupational exposome context.

Methods We have conducted a semi-systematic literature review to identify self-sampling techniques used to generate high quality data on several biomarkers of exposure and effect. We are exploring the possibility of using these self-sampling techniques through a pilot study. A tiered analytical approach along with a biological sequence will be followed to efficiently analyze the samples (i.e. blood, urine, saliva, exhaled breath, exhaled breath aerosols and exhaled breath condensate) for a broad spectrum of biomarkers and omics. Additionally, non-invasive targeted and non-targeted exposome markers of acute lung function decline and inflammation will be developed through proteomic analysis of exhaled breath condensate (EBC), and exhaled breath VOCs using the ReCIVA Breath Sampler. These data will be integrated to generate signatures or 'fingerprints' of exposomes, at individual and group levels.

Results and Conclusion The developed methodology will be applied in 2 cohorts within the EPHOR project: shift-workers and workers with asthma or allergic rhinitis to assess the internal exposure and elucidate biological pathways in disease development.

\section{S-236 SUICIDE AND JOB LOSS IN THE DIESEL EXHAUST IN MINERS STUDY II (DEMS II)}

'Jacqueline Ferguson, Ellen Eisen, Hilary Colbeth, Sadie Costello, Andreas Neophytou, Stella Koutros, Debra Silverman. 'Stanford University, United States

\subsection{6/OEM-2021-EPI.425}

Introduction The highest suicide rates in the U.S. have been found among males in construction, mining, and extraction occupations. In recent years, the rate has increased dramatically, which may reflect the lack of economic opportunities for miners.

Objectives We examined the impact of job loss, both permanent and temporary, on risk of suicide and overdose among a cohort of 11,817 male miners from the Diesel Exhaust in Miners Study II.

Methods We calculated directly standardized age-adjusted incidence rates per 100,000 person-years from 1947 through 2015 by calendar period. We fit Cox models to estimate hazard ratios (HRs) for suicide and overdose in relation to leaving work, age at leaving work, and intermittent time-off work. Separate models were fit for job loss pre- and post1980 to examine effect modification by time period as the industry curtailed its workforce in the early 1980s.

Results The age-adjusted incidence suicide/overdose death rate was 64.7 (95\% CI: 56.4, 73.9) per 100,000. Suicide deaths peaked between 1980 and 1989 at 67.2 before declining. Based on 248 suicides and overdoses, miners who left work were more likely to die by suicide/overdose compared to those remained at work (HR 1.59 (95\% CI: 1.10, 2.29)). Effect modification by decade was present as the HRs for suicide/ overdose and age at leaving work differed significantly between the stratified analyses (job loss pre-1980 vs. post1980). Among miners who left work prior to 1980, HRs for 